\title{
Experience: A Summer Elective in Germany
}

\author{
Maria Grazia Grech.
}

\section{The Experience}

About the Author: Maria Grazia Grech is a final-year medical student at the University of Malta Medical School, Msida, Malta.
In August of 2015, I underwent a month-long clinical elective at a Red Cross Hospital in Kassel, Germany. This hospital is relatively small compared to the major hospital in the same town, however, it deals with a varied case mix of patients across many disciplines including internal medicine, general surgery, trauma and orthopedics.

Prior to the experience, I was required to take German language lessons during the academic year to familiarize myself with the basics. Sometimes, I saw this as a nuisance, especially when I had other more pressing topics to revise for our medical curriculum, but I convinced myself that I had the added bonus of learning an extra language and put in the extra effort.

Although I have had a great interest in oncology since before starting medical school, it is unfortunate that we do not have any clinical attachments during our medical course in Malta. As such, this was an opportunity for me to broaden my horizons and to gain hands-on knowledge. My home country, Malta, is an extremely small island, so students continue to live with their families while attending university. Therefore, taking part in such an experience in Germany, away from my family, gave me the added opportunity of experiencing living alone. This helped me learn how to be more responsible, mature and self-disciplined.

The procedures I was involved in included ultrasounds, examination and follow-up of cancer patients, bone marrow aspirations and biopsies, and examining their histology, in addition to participating in ward rounds and interdisciplinary meetings. I saw people at different stages of illness; on one hand there were people who had just been diagnosed, and on the other there were patients undergoing palliative care. I also gained an insight into the difficulties of living with such illnesses. The treatments involved had a myriad of side-effects, and seeing patients going through those complications was a sobering experience. Coming face to face with death every day reminded me of the fragility and unpredictability of life, something which people working in professions that witness death regularly realize to0;' cancer does not discriminate between young or old, rich or poor, good or bad. It helped me appreciate what I have, and taught me to live in the moment.

Two patients stood out particularly for me. The first had been referred to hospice after his colon cancer had metastasized widely. Unfortunately, he passed away during the first week of my elective. For me, it was the first time I had ever witnessed a patient whom I was involved in treating die. Although we had previously had lectures and sessions on how to deal with death, the reality of it had a profound effect on me; I had known him for only a short while, but I still felt sad. Seeing his relatives reminded me that he was not just a patient, but also a loving husband, father, brother and uncle. Additionally, he was a Muslim resident of a predominantly Catholic country which had received a lot of recent immigrants from Muslim nations. This helped me to learn about traditions surrounding death of a loved one in a culture I was not familiar with, knowledge that I took back to my own country and used to help understand patients better.

The other patient who deeply affected me was also in palliative care, as he had prostate cancer which had metastasized to his vertebrae, as well as hidradenitis suppurativa, an excruciatingly painful condition in which most of one's body fills up with sores discharging pus. It was very hard to care for him because his condition was complex, but it reminded me to never neglect the humanity and dignity of a patient. This same patient taught me a lot about hope. After weeks of being bedridden, it was an exhilarating feeling to finally see him outside enjoying some sunshine.

Reflecting on this experience, I also learnt about protocols involved in treating cancer patients, as well as a lot about the ethics surrounding end-of-life decisions. Professionally, I observed the doctor-patient relationship from up-close and this allowed me to assimilate and learn how to utilize empathy and sound clinical reasoning in practice. I saw and gained knowledge in breaking bad news to patients, and how to deal with difficult people; a distraught patient in need of answers is a completely different arena than being taught a couple of sentences as part of a lecture. I learnt how important a multidisciplinary

Submission: Mar 11, 2016

Acceptance: Jul 05, 2016

Publication: Aug 31, 2016

${ }^{1}$ Faculty of Medicine and Surgery, University of Malta, Msida, Malta. 


\section{Experience}

approach is in treating patients since a lot of collaboration and consultations were needed between different specialties.

Malta and Germany both have a similar quality of life; the standard of living is high in both countries so it was easy to compare practices between countries, unlike students or doctors who come from developing countries. ${ }^{2}$ Like Malta, most patients we saw were elderly, with multiple comorbidities. Even diagnoses were similar, with breast, colon cancers and hematological malignancies being the commonest malignancies being treated. However, culturally, people were different. My main barrier was communication since in Germany, even medical staff were not always proficient in English and I only knew the basics of the German language. Apart from that, in Malta people tend to be openly religious whereas in Cermany people are subtler and also more secular. Therefore, I learnt to be careful about what I say to respect others' beliefs.

I now feel much better informed about deciding which specialty to choose in the future, as this gave me a first-hand experience in oncology. I am deeply grateful to the staff who made every effort possible to ensure I had the best visit possible, and who spent a lot of time teaching me new skills. In the end, writing this experience only made my passion for the specialty of oncology grow stronger. I would definitely recommend that students participate in similar clinical attachments. As William Osler once stated: "To study the phenomena of disease without books is to sail an uncharted sea, while to study books without patients is not to go to sea at all."

\section{References}

1. Chong ZX. Experience of a medical student: volunteering in the emergency department during massive flood in Kelantan, Malaysia. Int J Med Students. 2015 Jul 13;3(3):163-4.

2. Delgado-Hurtado JJ. An international medical graduate's experience of postgraduate training in the United States. Int J Med Students. 2015 Aug 14;3(3):165-6.

3. Osler W. Aequanimitas with other addresses to medical students, nurses and practitioners of medicine. 2nd ed. Philadelphia (PA): P. Blakiston's Son a C0; 1910. p. 220.

\footnotetext{
Acknowledgments

Special thanks to Rotes Kreuz Krankenhaus Gemeinnützige GmbH, Malta Medical Students' Association, the German-Maltese Circle in Malta, and the German Maltese Medical Society.
}

\section{Conflict of Interest Statement at Funding}

Grants were organized by the German Maltese Circle and the German Maltese Medical Society.

Author Contributions

Conceptualization, Writing: MGG.

Cite as:

Grech MG. Experience: a summer elective in Germany. Int J Med Students. 2016 May-Aug;4(2):78-9. 